\title{
Incidence and Characterization of Rice Root Nematodes, Hirschmanniella mucronata, from Rice Fields in Pathum Thani Province, Thailand
}

\author{
Natthidech Beesa ${ }^{1,3}$, Anongnuch Sasnarukkit ${ }^{1}$, Kansiree Jindapunnapat ${ }^{2}$, \\ Buncha Chinnasri ${ }^{1}$ and Thanunchanok Chairin ${ }^{3, *}$ \\ ${ }^{I}$ Department of Plant Pathology, Faculty of Agriculture, Kasetsart University, Bangkok 10400, Thailand \\ ${ }^{2}$ Department of Entomology and Plant Pathology, Faculty of Agriculture, Khon Kaen University, \\ Khon Kaen 40002, Thailand \\ ${ }^{3}$ Agricultural Innovation and Management Division (Pest Management), Faculty of Natural Resources, \\ Prince of Songkla University, Hat Yai, Songkhla 90110, Thailand
}

("Corresponding author's e-mail: thanunchanok.c@psu.ac.th)

Received: 15 August 2020, Revised: 30 April 2021, Accepted: 5 May 2021

\begin{abstract}
The rice root nematodes, Hirschmanniella oryzae and H. mucronata, are serious and widespread threats to global rice production, especially in tropical and subtropical zones. Reliable and efficient identification of these nematodes is vital to nematode control and management. In this current study, 36 soil or rice root samples were collected from rice fields located in Pathum Thani province and used for nematode extraction. The results demonstrated that Hirschmanniella sp. was found in all paddy fields, especially in Muang District, where RD47 rice cultivars were grown at soil pH of 5.9. Molecular identification based on the analysis of $28 \mathrm{~S}$ rRNA and 18S-ITS1-1.58S genes revealed $98-99 \%$ similarity to $H$. mucronata, and its phylogenetic trees were grouped with $H$. mucronata from Cambodia and Philippines. Likewise, the morphometric characterization revealed remarkable features of $H$. mucronata with long body $(1,639-2,329 \mu \mathrm{m})$, long stylet $(24-27 \mu \mathrm{m})$ and an obvious mucron at the end of terminus. Therefore, the main rice root nematode species found in Pathum Thani's rice fields were $H$. mucronata.
\end{abstract}

Keywords: Nematode identification, Hirschmanniella mucronata, Rice cultivars, Pathum Thani

\section{Introduction}

Rice (Oryza sativa L.) is a vital economic crop of Thailand; both for exportation and household consumption. In the central plain of Thailand, Pathum Thani province has been recorded as one of the most important places for a rice cultivation, with rice planting area of $55 \%$ of the total area and rice production of up to 213,238 tons per year [1,2]. Currently, the main challenges for rice cultivation include the depletion of soil fertility and plant pests due to the practice of continuous rice cultivation for a long time [3]. Numerous countries reported that one of the most serious pests in rice is plant-parasitic nematodes [4,5]. Hirschmanniella spp., rice root nematodes, is one of the most prevalent plant-parasitic nematodes that can be found up to $90 \%$ of paddy rice fields. Moreover, this nematode has a wide host range of more than 30 plant species [6,7]. The estimated rice yield losses caused by Hirschmanniella sp. around the world are approximately $25 \%$ [7]. One of the most predominant species of Hirschmanniella is H. oryzae, which causes stunting and up to $60 \%$ reduction in rice tillering. In most cases, the nematode population densities in soil are low and the below ground symptoms such as discoloration, deterioration, and rotting of rice roots are usually unnoticeable by the farmers. However, a decline in plant growth can happen and be readily observed by the experts [8]. In Asian countries, Hirschmanniella sp. is widely distributed and infects rice fields. This nematode was identified by molecular and morphological characters as $H$. oryzae and H. mucronata in China and Philippines, respectively $[9,10]$. Furthermore, H. mucronata was reported in Cambodia [11,20]. In recent years, Hirschmanniella spp. was reported in Nakhon Nayok's rice fields in Thailand [12]. Nevertheless, Bridge et al. [13] reported that $H$. oryzae was found in Thailand. Khun et al. [11] summarized that Hirschmanniella species may result in intraspecific variation when identification is conducted by a sole morphological analysis and this data should be substantiated by molecular 
characterization. Therefore, this study was conducted to examine the extent of infestation of Hirschmanniella and to identify the species of this nematode collected from Pathum Thani's rice fields by using molecular and morphological characteristics.

\section{Materials and methods}

\section{Soil and rice root sampling}

Thirty-six soil or rice root samples were collected from paddy rice fields of 6 districts in Pathum Thani province, including Nong Suea, Thanyaburi, Lam Luk Ka, Khlong Luang, Muang and Sam Khok ( 2 fields/district). In each field, 6 soil or root samples were randomly collected from the rice rhizosphere between $0-20 \mathrm{~cm}$ depth from the soil surface. After that, the collected soil or rice root samples were separately mixed and 3 sub-samples were chosen from this mixed soil or root samples to be represented (replications) of each field. These composite subsamples were put into plastic bags, labeled, and transported to the laboratory for nematode extraction. In addition, the field data, including rice cultivars and ages, soil $\mathrm{pH}$ (soil $\mathrm{pH}$ and moisture tester Takemura, Japan) and GPS position (The IOS 7 Compass app) were recorded.

\section{Nematode extraction}

Nematodes were extracted from $150 \mathrm{~g}$ soil by the Cobb's Sieving and Decantation and the Modified Baermann's Funnel techniques [14]. In brief, nematodes were extracted from soil by pouring the supernatants of soil suspensions into a series of 250, 105 and $37 \mu \mathrm{m}$ aperture sieve. Nematodes suspended on the 105 and $37 \mu \mathrm{m}$ mesh sieves were collected and placed on tissue papers lined on a wire screen that had been suspended on the funnels. Two days later, nematodes at the bottom of the funnel were collected, observed, and counted under a compound microscope (Olympus BX50, USA).

For rice root samples, the collected roots from each field were cut into about 1 -inch-long pieces and thoroughly mixed. Subsequently, $10 \mathrm{~g}$ of roots were randomly selected and used for nematode extraction. Root samples were washed free of soil with tap water, then cut into around $0.5-1 \mathrm{~cm}$ length and placed on the tissue papers lined on a plastic sieve in the tray. Later on, water was gently added into the tray until the roots were completely submerged [15]. Two days later, nematodes suspended in the tray were collected by $37 \mu \mathrm{m}$ mesh sieve, observed, and counted under a compound microscope (Olympus BX50, USA).

\section{Statistical analysis}

Data were statistically analyzed by the SPSS software (version 26.0; SPSS Inc.; Chicago, IL, USA). Differences in the densities of nematodes among all rice fields were determined by analysis of variance (ANOVA) and the means were compared using Duncan adjustment for multiple comparisons $(p \leq 0.05)$.

\section{Molecular identification}

Hirschmanniella collected from each district were used for DNA extraction and analysis by the polymerase chain reaction (PCR). Briefly, one adult Hirschmanniella was placed in a $0.5 \mathrm{~mL}$ PCR tube filled with $25 \mu \mathrm{L}$ of distilled water. Then, $25 \mu \mathrm{L}$ of lysis buffer [200 mM NaCl (A\&D Technology, Japan), $200 \mathrm{mM}$ Tris-HCl pH 8.0 (A\&D Technology, Japan), 1 \% (v/v) $\beta$-mercaptoethanol (Sigma, Japan), and $800 \mu \mathrm{g} / \mathrm{mL}$ proteinase $\mathrm{K}$ (Worthington Biochemical, USA)] was added into the tube. The reaction was incubated for $90 \mathrm{~min}$ at $65^{\circ} \mathrm{C}$, followed by $5 \mathrm{~min}$ at $99{ }^{\circ} \mathrm{C}$ in a PCR machine (Biometra Tgradient Thermoblock PCR Thermocycler, UK). Later, the extracted DNA was stored at $-20{ }^{\circ} \mathrm{C}$ until used as a DNA template [16].

The PCR was conducted by using extracted nematode DNA as the template. $30 \mu \mathrm{L}$ of PCR reaction included $3 \mu \mathrm{L}$ of DNA template, $9 \mu \mathrm{L}$ of sterilized distilled water, $1.5 \mu \mathrm{L}$ of each $10 \mu \mathrm{M}$ forward and reverse primers \{D2A (5' ACAAGTACCGTGAGGGAAAGT 3') and D3B (5' TGCGAAGGAACCAGCTACTA 3') [17] and rDNA2 (5'-TTGATTACGTCCCTGCCCTTT-3') and rDNA1.58s (5'-ACGAGCCCGAGTGATCCACCG-3') [18]\}, and $15 \mu \mathrm{L}$ of 2x PCR master mix with dye solution i-taq (Intron biotechnology, Korea). The PCR condition was programmed as follows: denaturation at $94{ }^{\circ} \mathrm{C}$ for $5 \mathrm{~min}$, followed by 35 cycles of $94{ }^{\circ} \mathrm{C}$ for $30 \mathrm{~s}, 56{ }^{\circ} \mathrm{C}$ for $30 \mathrm{~s}$ and $72{ }^{\circ} \mathrm{C}$ for 1 min, and final extension at $72{ }^{\circ} \mathrm{C}$ for $5 \mathrm{~min}$. The PCR products were loaded into $1.5 \%$ agarose buffered gels in 1xTAE buffer. The band size of PCR products was compared with 100+bp DNA marker (Biotechrabbit, Germany). The gel was run for $25 \mathrm{~min}$ at $100 \mathrm{~V}$ and visualized on an UV box. The PCR products were purified and submitted for sequencing at the SOLGEN Inc., Korea. Then, the DNA 
sequences were compared with GenBank in the National Center for Biotechnology Information (NCBI), available online from https://www.ncbi.nlm.nih.gov.

Phylogenetic trees were constructed by the Molecular Evolutionary Genetics Analysis version 7.0 using the DNA sequences at 28S and 18S-ITS1-5.8S gene regions of Hirschmanniella obtained from this study. DNA sequences of plant-parasitic nematodes associated with rice were selected from GenBank. Consequently, the alignments between these GenBank nucleotide sequences with those generated from each primer set were done using ClustalW. Phylogenetic trees were performed via the Maximum Likelihood (ML) methods based on Gamma distribution $(\mathrm{GTR}+\mathrm{G})$ model and the test of phylogeny done by the rapid bootstrap algorithm (1000 iterations) $[19,20]$.

\section{Morphological identification}

A total of 24 Hirschmanniella males and females (4 nematodes/district) were killed by hot water at $50{ }^{\circ} \mathrm{C}$ and mounted on a drop of distilled water on a glass slide. Then, nematodes were observed and pictured by a digital camera (Canon Power Shot A640), which had been equipped with EOS Utility program and mounted on the compound microscope (Olympus BX50). Sizes of nematodes were measured via Axio Vision SE64 Rel. 4.9.1 program, and finally compared with the polytomous key [11]. The morphometrics were then calculated as follows: $\mathrm{L}=$ Total body length, $\mathrm{a}=$ Body length/Body width, $\mathrm{b}=$ Body length/Anterior end to pharyngo-intestinal junction (PIJ), $\mathrm{b}^{\prime}=$ Body length/Pharynx length, $\mathrm{c}=$ Body length/Tail length, $\mathrm{c}^{\prime}=$ Tail length/Maximum tail width, $\mathrm{V} \%=$ Head to vulva length/Body length $\times 100$, Stylet length, Maximum body width, Pharynx length, Anterior end to PIJ, Head to vulva length, Maximum tail width, and Tail length [11].

\section{Results and discussion}

\section{Incidence of Hirschmanniella in Pathum Thani's rice fields}

Hirschmanniella was found in all surveyed rice fields in Pathum Thani province (Table 1). In addition, more abundance of Hirschmanniella was observed in rice roots than in soil. Highest numbers of nematodes (1,214.6 nematodes/10 g roots) were significantly evident in Muang district no. 1 where RD47 rice cultivar was grown, followed by Sam Khok district no.1 and no.2 (992 and 772.3 nematodes/10 g roots, respectively; RD31 and Pathum Thani 1 cultivars, respectively). However, lowest numbers of nematodes were found in Nong Suea district no.2 and Muang district no. 2 (79.3 and 136 nematodes/10 g roots; RD49 and RD14 cultivars, respectively). The age of rice and soil $\mathrm{pH}$ might influence nematode abundance, where greater numbers of nematodes extracted from rice stubbles were observed at soil $\mathrm{pH}$ 5.9 - 6.0 than soil $\mathrm{pH} 5.3$ - 5.4. These results were similar to those reports by $[11,18,20]$ who examined Hirschmanniella populations in paddy rice fields of Takeo, Battambang and Kampong Thom provinces, Cambodia. In addition, as Hirschmanniella are migratory endoparasites by nature, they are more prevalent in rice roots than in soil [21]. Burns [22] mentioned that Hoplolaimus galeatus and members of the Tylenchinae-Psilenchinae survived the best at $\mathrm{pH}$ 6.0. This information reflects on the results of our experiments, where greater numbers of nematodes were found in rice roots than in soil and the highest density of nematodes was found at pH 5.9 in Muang district's rice field.

Table 1 Incidence and density of Hirschmanniella spp. on various rice cultivars and rice fields from Pathum Thani province.

\begin{tabular}{|c|c|c|c|c|c|}
\hline \multirow{2}{*}{ Districts } & \multirow{2}{*}{ Location (GPS) } & \multirow{2}{*}{$\begin{array}{c}\text { Rice varieties } \\
\text { (Age) }\end{array}$} & \multirow{2}{*}{ Soil pH } & \multicolumn{2}{|c|}{ Number of nematodes } \\
\hline & & & & 150 g soil & $10 \mathrm{~g}$ root \\
\hline Nong Suea 1 & $\begin{array}{c}14^{\circ} 6^{\prime} 39^{\prime \prime N} \\
100^{\circ} 50^{\prime} 45^{\prime \prime} \mathrm{E}\end{array}$ & $\begin{array}{c}\text { RD47 } \\
\text { (110 days) }\end{array}$ & 5.9 & $8.3 \pm 2.6 \mathrm{~cd}^{/ 1}$ & $190.6 \pm 21.6 \mathrm{e}$ \\
\hline Nong Suea 2 & $\begin{array}{c}14^{\circ} 6^{\prime} 46^{\prime \prime N} \\
100^{\circ} 50^{\prime} 31 " \mathrm{E}\end{array}$ & $\begin{array}{c}\text { RD49 } \\
\text { (110 days) }\end{array}$ & 5.8 & $2.3 \pm 0.3 \mathrm{~cd}$ & $79.3 \pm 13.3 \mathrm{f}$ \\
\hline Thanyaburi 1 & $\begin{array}{c}14^{\circ} 2 ' 28^{\prime \prime} \mathrm{N} \\
100^{\circ} 49^{\prime} 27^{\prime \prime} \mathrm{E}\end{array}$ & $\begin{array}{l}\text { Phitsanulok } 2 \\
\text { (rice stubble) }\end{array}$ & 5.4 & $5 \pm 1.2 \mathrm{~cd}$ & $316.6 \pm 18.1 \mathrm{~d}$ \\
\hline Thanyaburi 2 & $\begin{array}{c}14^{\circ} 2^{\prime} 29^{\prime \prime} \mathrm{N} \\
100^{\circ} 49^{\prime} 28^{\prime \prime} \mathrm{E}\end{array}$ & $\begin{array}{l}\text { Phitsanulok } 2 \\
\text { (rice stubble) }\end{array}$ & 5.3 & $8 \pm 1.7 \mathrm{~cd}$ & $199 \pm 16.2 \mathrm{e}$ \\
\hline Lam Luk Ka 1 & $\begin{array}{c}14^{\circ} 1^{\prime} 42^{\prime \prime N} \\
100^{\circ} 49^{\prime} 28^{\prime \prime} \mathrm{E}\end{array}$ & $\begin{array}{l}\text { Pathum Thani } 1 \\
\text { (rice stubble) }\end{array}$ & 5.3 & $4.6 \pm 1.5 \mathrm{~cd}$ & $159 \pm 4 \mathrm{ef}$ \\
\hline Lam Luk Ka 2 & $\begin{array}{c}14^{\circ} 1^{\prime} 41^{\prime \prime} \mathrm{N} \\
100^{\circ} 49^{\prime} 32^{\prime \prime} \mathrm{E}\end{array}$ & $\begin{array}{l}\text { Pathum Thani } 1 \\
\text { (rice stubble) }\end{array}$ & 5.3 & $43.3 \pm 7.2 \mathrm{a}$ & $330 \pm 13.85 \mathrm{~d}$ \\
\hline
\end{tabular}




\begin{tabular}{|c|c|c|c|c|c|}
\hline \multirow{2}{*}{ Districts } & \multirow{2}{*}{ Location (GPS) } & \multirow{2}{*}{$\begin{array}{c}\text { Rice varieties } \\
\text { (Age) }\end{array}$} & \multirow{2}{*}{ Soil pH } & \multicolumn{2}{|c|}{ Number of nematodes } \\
\hline & & & & 150 g soil & $10 \mathrm{~g}$ root \\
\hline Khlong Luang 1 & $\begin{array}{c}14^{\circ} 5^{\prime} 35^{\prime \prime} \mathrm{N} \\
100^{\circ} 38^{\prime} 31 " \mathrm{E}\end{array}$ & $\begin{array}{c}\mathrm{RD} 41 \\
\text { (rice stubble) }\end{array}$ & 5.6 & $11 \pm 2.9 \mathrm{c}$ & $313 \pm 12.7 d$ \\
\hline Khlong Luang 2 & $\begin{array}{c}14^{\circ} 5^{\prime} 38^{\prime \prime} \mathrm{N} \\
100^{\circ} 38^{\prime} 28^{\prime \prime} \mathrm{E}\end{array}$ & $\begin{array}{c}\mathrm{RD} 41 \\
\text { (rice stubble) }\end{array}$ & 5.5 & $44.3 \pm 3.7 \mathrm{a}$ & $349.3 \pm 29.7 d$ \\
\hline Muang district 1 & $\begin{array}{c}14^{\circ} 2^{\prime} 8^{\prime \prime} \mathrm{N} \\
100^{\circ} 30^{\prime} 19^{\prime \prime} \mathrm{E}\end{array}$ & $\begin{array}{l}\mathrm{RD} 47 \\
\text { (rice stubble) }\end{array}$ & 5.9 & $34 \pm 3.5 b$ & $1,214.6 \pm 97.8 \mathrm{a}$ \\
\hline Muang district 2 & $\begin{array}{c}14^{\circ} 2^{\prime} 1 " \mathrm{~N} \\
100^{\circ} 30^{\prime} 55^{\prime \prime} \mathrm{E}\end{array}$ & $\begin{array}{c}\mathrm{RD} 14 \\
\text { (rice stubble) }\end{array}$ & 6.0 & $2 \pm 0.5 \mathrm{~cd}$ & $136 \pm 32.3 \mathrm{ef}$ \\
\hline Sam Khok 1 & $\begin{array}{c}14^{\circ} 6^{\prime} 41^{\prime \prime N} \\
100^{\circ} 33^{\prime} 53^{\prime \prime} \mathrm{E}\end{array}$ & $\begin{array}{c}\text { RD31 } \\
\text { (60 days) }\end{array}$ & 6.4 & $2.33 \pm 0.3 \mathrm{~cd}$ & $992 \pm 13.85 b$ \\
\hline Sam Khok 2 & $\begin{array}{c}14^{\circ} 6^{\prime} 40^{\prime \prime} \mathrm{N} \\
100^{\circ} 33^{\prime} 38^{\prime \prime} \mathrm{E}\end{array}$ & $\begin{array}{l}\text { Pathum Thani } 1 \\
\text { (rice stubble) }\end{array}$ & 6.7 & $0.33 \pm 0.3 \mathrm{~d}$ & $772.3 \pm 17.7 \mathrm{c}$ \\
\hline
\end{tabular}

${ }^{1 /}$ mean of the numbers of Hirschmanniella \pm standard error $(\mathrm{n}=3)$ were compared using Duncan adjustment for multiple comparisons $(p<0.05)$. Similar lower-case letters in each column indicated that means are not significantly different.

\section{Molecular identification}

DNA fragments of Hirschmanniella, amplified by D2A/D3B and rDNA2/rDNA1.58s, showed the size of 750 and $470 \mathrm{bp}$, respectively (Figure 1). Consequently, a comparison of nucleotide sequences based on 28S rRNA (GenBank accession no. MT597911-MT597916) and 18S-ITS1-5.8S (MT60262 MT602633) gene regions (Table 2) showed high homology with H. mucronata (more than $98-99 \%$ similarity) from Cambodia, Philippines, Taiwan and China. This result coincided with reports by Berg et al. [17] who reported the length of amplicon D2-D3 of 28S rRNA and partial 18S rRNA for Hirschmanniella spp. of 650 - $800 \mathrm{bp}$. On the contrary, [20] reported that the size of DNA fragments amplified by rDNA2/rDNA1.58s was $470 \mathrm{bp}$.

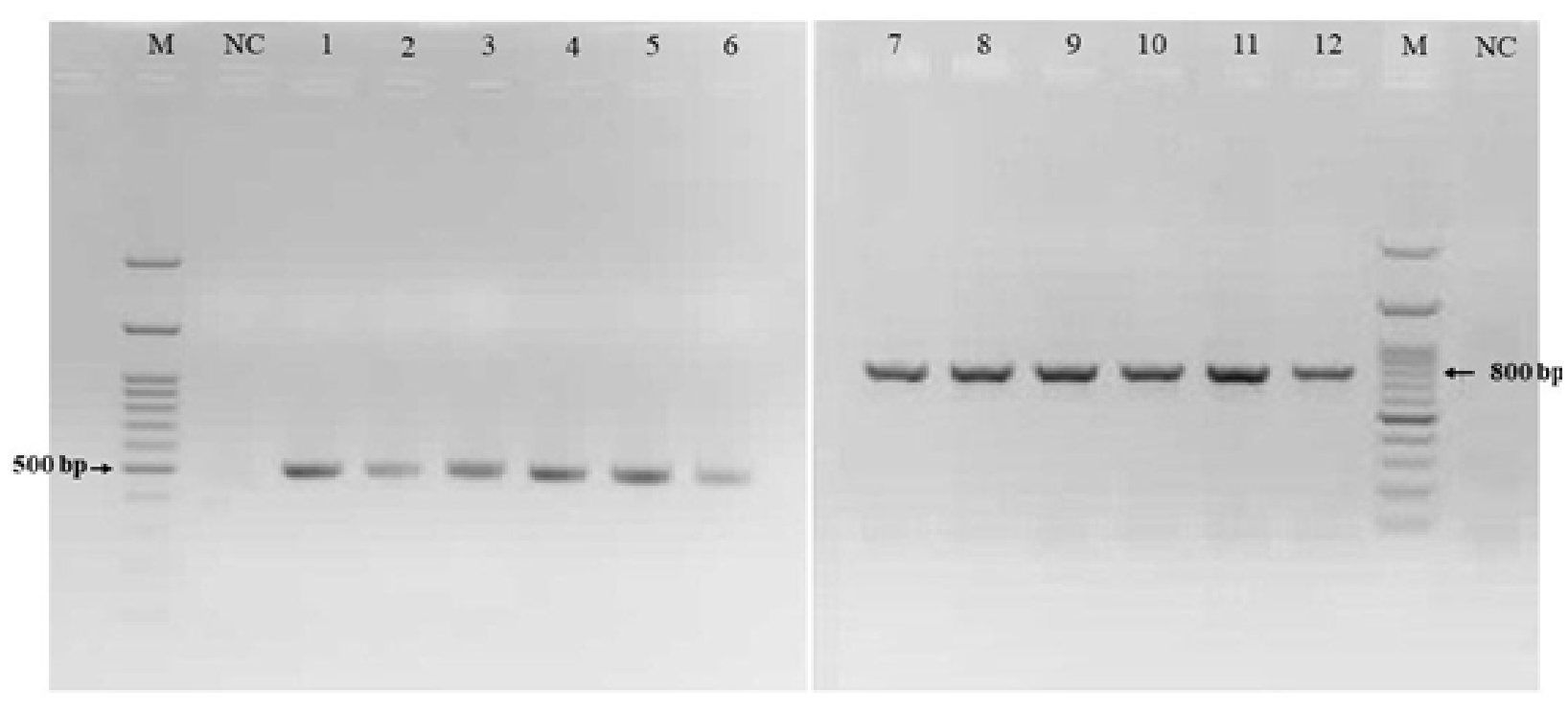

Figure 1 Agarose gel electrophoresis of PCR-amplified DNA of Hirschmanniella mucronata by using primers rDNA2/rDNA1.58s (Lane 1 - 6) and D2A/D3B (Lane 7 - 12). M: 100+bp DNA leader, NC: negative control, Lane 1,7: Nong Suea's sample, Lane 2,8: Thanyaburi's sample, Lane 3,9: Lam Luk Ka's sample, Lane 4,10: Khlong Luang's sample, Lane 5,11: Muang district's sample, Lane 6,12: Sam Khok's sample. 
Table 2 List of new GenBank accession numbers.

\begin{tabular}{|c|c|c|c|c|}
\hline Nematode species & isolate & Location & Sequenced region & $\begin{array}{c}\text { GenBank } \\
\text { accession number }\end{array}$ \\
\hline Hirschmanniella mucronata & $\mathrm{PN}$ & Nong Suea & $28 \mathrm{~S}$ & MT597911 \\
\hline H. mucronata & PT & Thanyaburi & $28 \mathrm{~S}$ & MT597912 \\
\hline H. mucronata & PL & Lam Luk Ka & $28 \mathrm{~S}$ & MT597913 \\
\hline H. mucronata & PK & Khlong Luang & $28 \mathrm{~S}$ & MT597914 \\
\hline H. mucronata & PM & Muang district & $28 \mathrm{~S}$ & MT597915 \\
\hline H. mucronata & PS & Sam Khok & $28 \mathrm{~S}$ & MT597916 \\
\hline H. mucronata & $\mathrm{PN}$ & Nong Suea & $18 \mathrm{~S}-\mathrm{ITS} 1-5.8 \mathrm{~S}$ & MT602628 \\
\hline H. mucronata & PT & Thanyaburi & 18S-ITS1-5.8S & MT602629 \\
\hline H. mucronata & PL & Lam Luk Ka & 18S-ITS1-5.8S & MT602630 \\
\hline H. mucronata & PK & Khlong Luang & 18S-ITS1-5.8S & MT602631 \\
\hline H. mucronata & PM & Muang district & $18 \mathrm{~S}-\mathrm{ITS} 1-5.8 \mathrm{~S}$ & MT602632 \\
\hline H. mucronata & PS & Sam Khok & 18S-ITS1-5.8S & MT602633 \\
\hline
\end{tabular}

The phylogenetic tree showed similarity between 28S rRNA and 18S-ITS1-5.8S gene regions (Figure 2 and 3), where the nematodes were identified as $H$. mucronata at bootstrap values $>98$ and related to $H$. kwazuna and $H$. loofi sisters, while $H$. oryzae were grouped in a different clade with low support. This result was similar to $[11,20]$, who reported that $H$. mucronata was relative to H. kwazuna and $H$. loofi sisters, with the single nucleotide polymorphisms (SNPs) at 28S region differed from $H$. loofi and $H$. kwazuna by $7.7-8.3$ and $8.1-8.7 \%$, respectively, while at ITS1-5.8S-ITS2 by $22.8-23.4$ and $22.7-23 \%$, respectively.

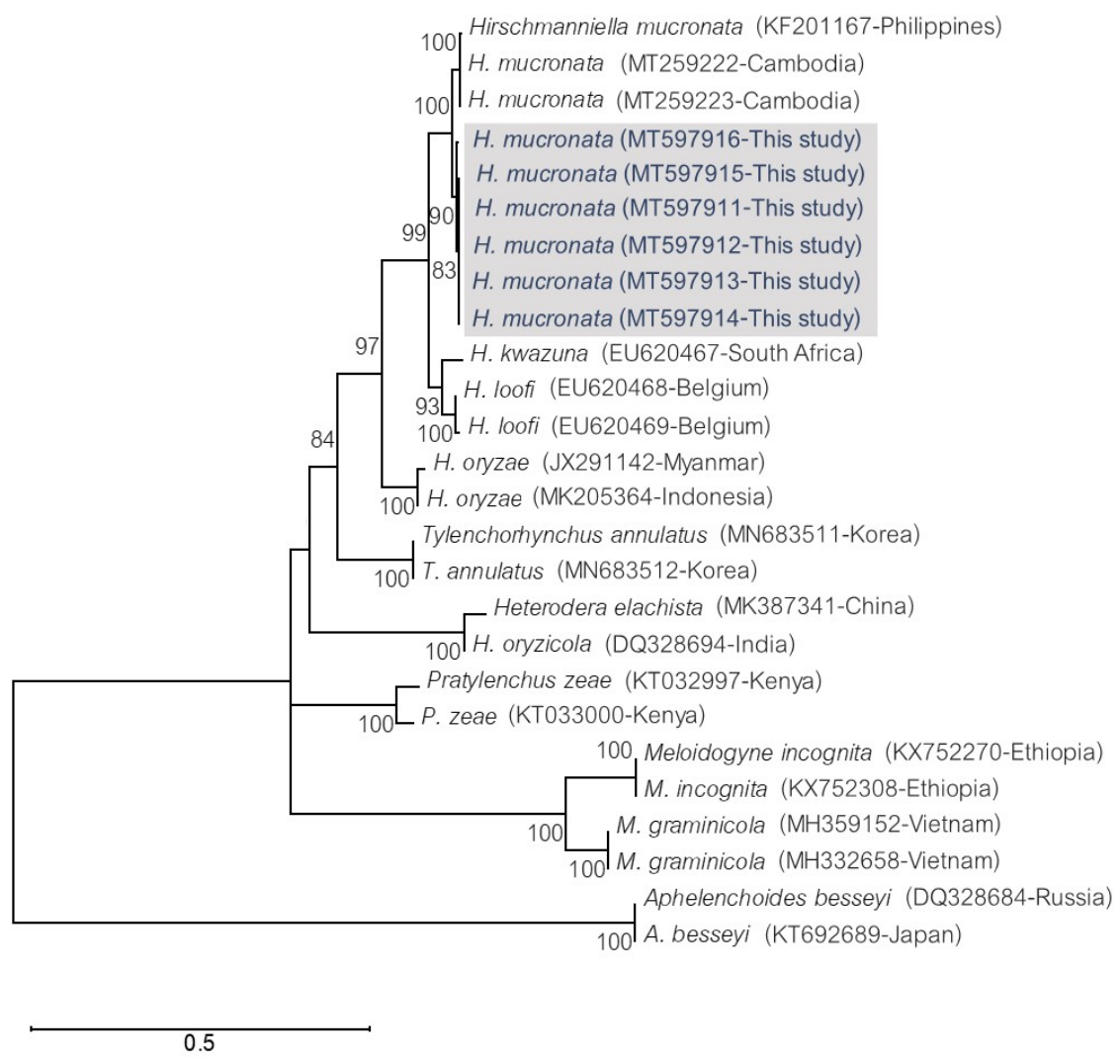

Figure 2 The phylogenetic tree of Hirschmanniella mucronata collected from Pathum Thani's rice fields and some other plant-parasitic nematodes in rice based on 28S rRNA gene. Maximum likelihood method was used to create a bootstrap consensus tree inferred from 1,000 replicates. Numbers beside branches represent ML bootstrap support values $\geq 70 \%$. Scale bar represents substitutions per nucleotide position. NCBI accession numbers are listed behind the species names. 


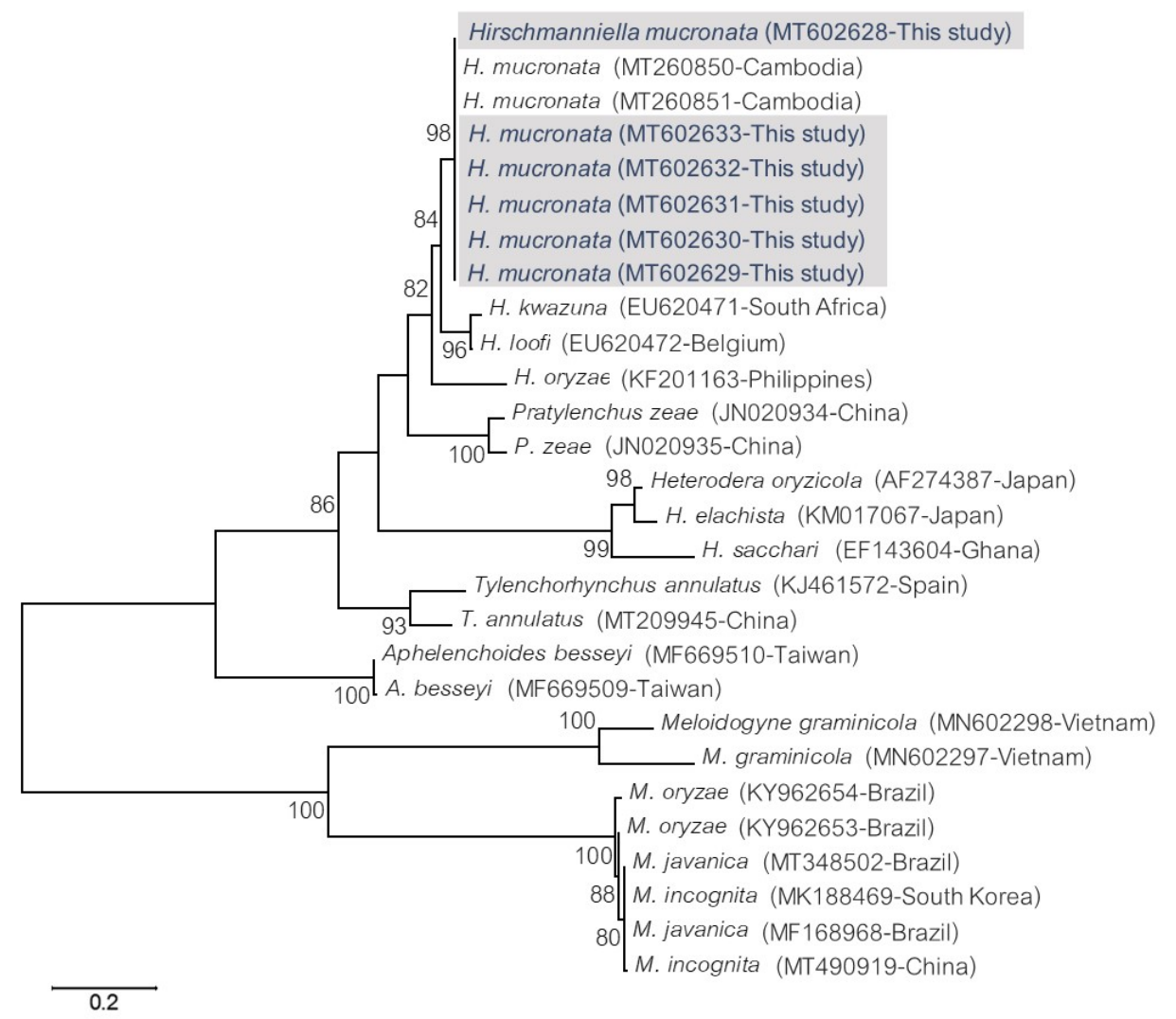

Figure 3 The phylogenetic tree of Hirschmanniella mucronata collected from Pathum Thani's rice fields and some other plant-parasitic nematodes in rice based on 18S-ITS1-5.8S genes. Maximum likelihood method was used to create a bootstrap consensus tree inferred from 1,000 replicates. Numbers beside branches represent ML bootstrap support values $\geq 70 \%$. Scale bar represents substitutions per nucleotide position. NCBI accession numbers are listed behind the species names.

\section{Morphological identification}

Morphological characteristics of Hirschmanniella were similar between males and females (Figure 4 and 5); Body length ranged from 1,639 - 2,329 $\mu \mathrm{m}$, stylet length $24-27 \mu \mathrm{m}$, basal knob round, distinctly offset, values of anterior end to pharyngo-intestinal junction (PIJ) $105-147 \mu \mathrm{m}$, long overlapping of esophagus over intestine, pharyngeal glands elongated, pharynx lengths $217-431 \mu \mathrm{m}$, tail lengths $65-108 \mu \mathrm{m}$, maximum tail widths $15-25 \mu \mathrm{m}$ and the end of tails terminal an obvious mucron. For females, the position of the vulva was located approximately $49.2-57.3 \%$ of body length with 2 ovaries, while Hirschmanniella male tail was possessed with a distinct bursa and spicules of $29-36 \mu \mathrm{m}$ long. Morphometric characteristics were as follows; $\mathrm{L}=1,639-2,329 \mu \mathrm{m}, \mathrm{V}(\%)=49.2-57.3, \mathrm{a}=52.4$ $72.8, \mathrm{~b}=12.4-19, \mathrm{~b}^{\prime}=4.2-8.7, \mathrm{c}=17.5-30$ and $\mathrm{c}^{\prime}=3.1-6$ (Table 3). These characteristics were matched with $H$. mucronata, which $[11,20]$ reported in Battambang and Takeo provinces, Cambodia. Although this study found $H$. mucronata, Bridge et al. [13] reported the occurrence of $H$. oryzae in Thailand. The difference between $H$. oryzae and $H$. mucronata includes shorter body length $(1,090$ $1,330 \mu \mathrm{m})$ and stylet length $(15-17 \mu \mathrm{m})$ of $H$. oryzae than those of $H$. mucronata [9]. 
Table 3 Morphometrics of Hirschmanniella mucronata isolated from Pathum Thani province and their comparison with those of the report from Takeo, Cambodia.

\begin{tabular}{|c|c|c|c|c|}
\hline \multirow[t]{2}{*}{ Character } & \multicolumn{2}{|c|}{$\begin{array}{c}\text { Hirschmanniella mucronata } \\
\text { (This study) }\end{array}$} & \multicolumn{2}{|c|}{$\begin{array}{c}\text { Takeo, Cambodia } \\
\text { H. mucronata } \\
{[11]}\end{array}$} \\
\hline & Female & Male & Female & Male \\
\hline $\mathrm{n}$ & 24 & 24 & 30 & 21 \\
\hline $\mathrm{L}$ & $\begin{array}{c}2,023 \pm 140 \\
(2,329-1,779)\end{array}$ & $\begin{array}{c}1,864 \pm 131 \\
(2,221-1,639)\end{array}$ & $\begin{array}{c}1,775 \pm 188 \\
(2,160-1,260)\end{array}$ & $\begin{array}{c}1,734 \pm 186 \\
(2,109-1,421)\end{array}$ \\
\hline $\mathrm{V}(\%)$ & $\begin{array}{c}53 \pm 1.7 \\
(57.3-49.2) \\
\end{array}$ & - & $\begin{array}{c}52 \pm 2.3 \\
(59-49)\end{array}$ & - \\
\hline $\mathrm{a}$ & $\begin{array}{c}63.7 \pm 4.2 \\
(72.8-55.8) \\
\end{array}$ & $\begin{array}{c}63.8 \pm 6 \\
(75.5-52.4) \\
\end{array}$ & $\begin{array}{c}58 \pm 5.2 \\
(67-47) \\
\end{array}$ & $\begin{array}{c}59 \pm 7.6 \\
(81-45) \\
\end{array}$ \\
\hline $\mathrm{b}$ & $\begin{array}{c}16 \pm 1.2 \\
(19-14) \\
\end{array}$ & $\begin{array}{c}14.5 \pm 1.4 \\
(18.5-12.4)\end{array}$ & $\begin{array}{c}14 \pm 1.1 \\
(16-12)\end{array}$ & $\begin{array}{c}13.9 \pm 1.7 \\
(16.7-11)\end{array}$ \\
\hline$b^{\prime}$ & $\begin{array}{c}6.1 \pm 1.1 \\
(8.7-4.8)\end{array}$ & $\begin{array}{c}5.5 \pm 0.6 \\
(7-4.2) \\
\end{array}$ & $\begin{array}{c}5.9 \pm 0.7 \\
(7.4-4.4)\end{array}$ & $\begin{array}{l}5.8 \pm 0.7 \\
(7-4.5)\end{array}$ \\
\hline $\mathrm{c}$ & $\begin{array}{c}23.6 \pm 3 \\
(30-18) \\
\end{array}$ & $\begin{array}{c}22.3 \pm 2.3 \\
(26.7-17.5)\end{array}$ & $\begin{array}{c}22 \pm 2.7 \\
(28-16) \\
\end{array}$ & $\begin{array}{c}21 \pm 2.2 \\
(25-17)\end{array}$ \\
\hline$c^{\prime}$ & $\begin{array}{c}4 \pm 0.4 \\
(4.6-3.1)\end{array}$ & $\begin{array}{l}4.5 \pm 0.6 \\
(6-3.6)\end{array}$ & $\begin{array}{l}3.7 \pm 0.4 \\
(5-2.8)\end{array}$ & $\begin{array}{c}4.4 \pm 0.4 \\
(5.1-3.5)\end{array}$ \\
\hline Stylet length & $\begin{array}{l}25.8 \pm 1^{/ 1} \\
(27-24)\end{array}$ & $\begin{array}{l}25.7 \pm 1.1 \\
(27-24)\end{array}$ & $\begin{array}{l}22.2 \pm 0.6 \\
(23-21)\end{array}$ & $\begin{array}{c}23 \pm 1.2 \\
(26-21)\end{array}$ \\
\hline Maximum body width & $\begin{array}{l}31.8 \pm 2.2 \\
(35-27)\end{array}$ & $\begin{array}{l}29.4 \pm 2.5 \\
(34-23)\end{array}$ & $\begin{array}{l}30.5 \pm 2.3 \\
(35-25)\end{array}$ & $\begin{array}{c}30 \pm 3.6 \\
(35-22)\end{array}$ \\
\hline Pharynx length & $\begin{array}{l}338.4 \pm 51.2 \\
(417-217)\end{array}$ & $\begin{array}{l}341.4 \pm 39.1 \\
(431-263)\end{array}$ & $\begin{array}{c}300 \pm 40 \\
(399-229)\end{array}$ & $\begin{array}{c}299 \pm 38 \\
(371-215)\end{array}$ \\
\hline Anterior end to PIJ & $\begin{array}{c}127.7 \pm 5.5 \\
(140-117)\end{array}$ & $\begin{array}{l}128.9 \pm 10.5 \\
(147-105)\end{array}$ & $\begin{array}{c}124 \pm 12 \\
(147-84)\end{array}$ & $\begin{array}{c}125 \pm 10.9 \\
(144-108)\end{array}$ \\
\hline Anterior to vulva length & $\begin{array}{l}1,077.3 \pm 65.5 \\
(1,188-958) \\
\end{array}$ & - & $\begin{array}{c}936 \pm 104 \\
(1,160-630) \\
\end{array}$ & - \\
\hline Spicule length & - & $\begin{array}{l}31.9 \pm 1.9 \\
(36-29)\end{array}$ & - & $\begin{array}{c}34 \pm 1.6 \\
(37-31) \\
\end{array}$ \\
\hline Maximum tail width & $\begin{array}{l}21.8 \pm 1.8 \\
(25-19)\end{array}$ & $\begin{array}{l}18.9 \pm 2.1 \\
(22-15)\end{array}$ & $\begin{array}{c}22 \pm 2.3 \\
(27-18)\end{array}$ & $\begin{array}{c}18.5 \pm 1 \\
(20-16)\end{array}$ \\
\hline Tail length & $\begin{array}{c}86.5 \pm 7.9 \\
(102-74) \\
\end{array}$ & $\begin{array}{c}84.3 \pm 9 \\
(108-65)\end{array}$ & $\begin{array}{c}81 \pm 8.2 \\
(99-60)\end{array}$ & $\begin{array}{l}82.6 \pm 9.9 \\
(98-63)\end{array}$ \\
\hline
\end{tabular}

${ }^{/ 1}$ All measurements are in $\mu \mathrm{m}$ and in the form: Mean \pm S.D. (Max-Min). 


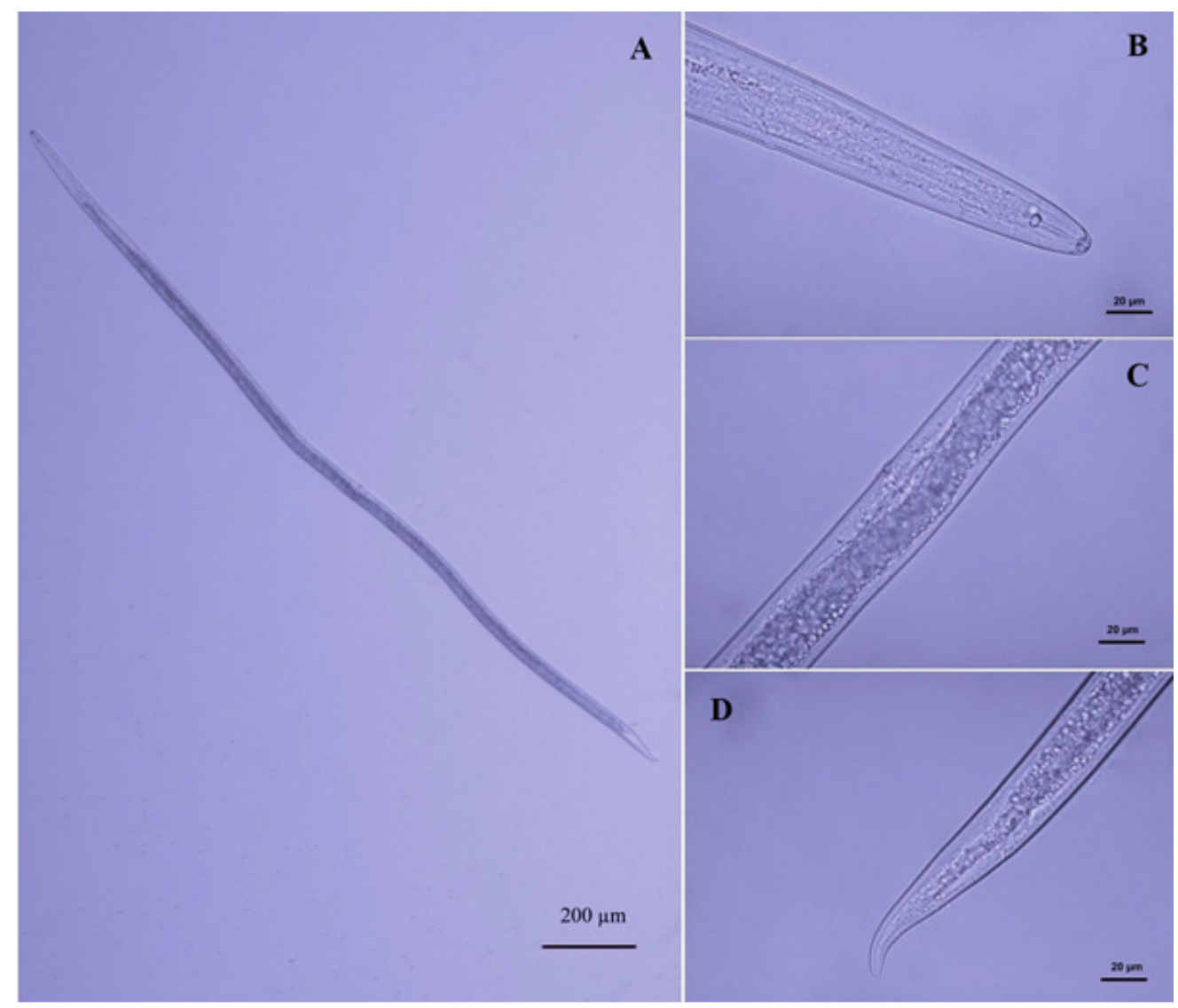

Figure 4 Photographs of Hirschmanniella mucronata female collected from paddy rice fields of Pathum Thani province, Thailand. A) Whole body $(100 \times)$, B) Anterior region $(1000 \times)$, C) Middle region $(1000 \times)$, D) Female tail $(1000 \times)$.

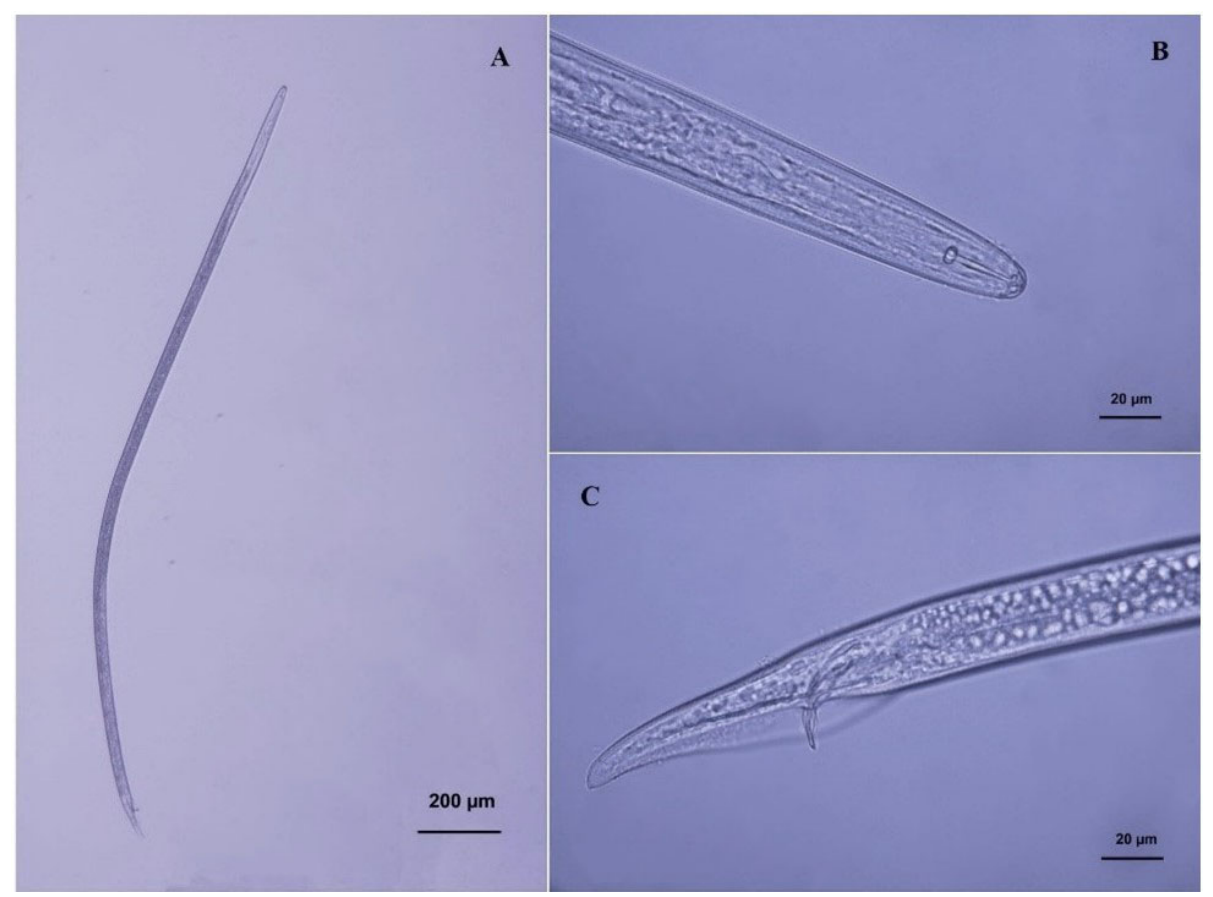

Figure 5 Photographs of Hirschmanniella mucronata male collected from paddy rice fields of Pathum Thani province, Thailand. A) Whole body $(100 \times)$, B) Anterior region $(1000 \times)$, C) Male tail $(1000 \times)$. 


\section{Conclusions}

This study demonstrated that Hirschmanniella infested all surveyed rice cultivars and all rice fields located in 6 districts of Pathum Thani province. The highest numbers of nematodes were found in RD47, followed by RD31 and Pathum Thani 1 cultivars, respectively. In addition, soil $\mathrm{pH}$ affected the number of nematodes, where higher nematode densities were evident at soil pH $5.9-6.0$ than $\mathrm{pH} 5.3$ - 5.4. The molecular characteristics based on $28 \mathrm{~S}$ and 18S-ITS1-5.8S genes revealed 98 - $99 \%$ similarity to H. mucronata and its phylogenetic trees were grouped with $H$. mucronata from Cambodia, Philippines, and China. Based on nematode morphology, long body $(1,639-2,329 \mu \mathrm{m})$, long stylet $(24-27 \mu \mathrm{m})$ and obvious mucron at the end of terminus were matched with those of $H$. mucronata. Therefore, the main rice root nematode species found in Pathum Thani's rice fields was $H$. mucronata. Further studies are needed, especially to determine the susceptibility of Thai local cultivars to this nematode.

\section{Acknowledgements}

The authors extend their gratitude to the Department of Plant Pathology, Faculty of Agriculture, Kasetsart University and Agricultural Innovation and Management Division (Pest Management), Faculty of Natural Resources, Prince of Songkla University, Thailand. This study was partially funded by the Participatory and Integrative Support for Agricultural Initiative (PISAI) Project ERASMUS +-Capacity Building in Higher Education Programme of the European Union for the support of the mobility of the double master's programme activities. This PISAI-E+ project is the international collaborative platform between 4 Thai higher education institutes (Prince of Songkla University, Chiang Mai University, Khon Kaen University and Kasetsart University) and European HEIs (SupAgro in France, Czech University of Life Science Prague in Czech Republic, University of Copenhagen in Denmark, University of Helsinki in Finland and Agrinatura.

\section{References}

[1] Rice Department, Available at: http://brrd.ricethailand.go.th/ricemap/riceCD52/index.phpurl=detail.php\&region_id=1\&province_id=13.htm, accessed July 2020.

[2] Office of Agricultural Economics, Available at: http://www.oae.go.th/assets/portals/1/fileups /prcaidata/files/second\%20rice\%2062\%20province\%2030-1-63-2(2).pdf, accessed January 2020.

[3] IJ Shelley, M Takahashi-Nosaka, M Kano-Nakata, MS Haque and Y Inukai. Rice cultivation in Bangladesh: present scenario, problems, and prospects. J. Int. Cooperat. Agr. Dev. 2016; 14, 20-9.

[4] JT Jones, A Haegeman, EGJ Danchin, HS Gaur, J Helder, MGK Jones, T Kikuchi, R ManzanillaLopez, JE Palomares-Rius, WML Wesemael and RN Perry. Top 10 plant-parasitic nematodes in molecular plant pathology. Mol. Plant Pathol. 2013; 14, 946-61.

[5] GC Bernard, M Egnin and C Bonsi. The impact of plant-parasitic nematodes on agriculture and methods of control. In: MM Shah and M Mohamood (Eds.). Nematology - concepts, diagnosis and control. InTech, London, 2017, p. 121-51.

[6] ZTZ Maung, PP Kyi, YY Myint, T Lwin and D Waele. Occurrence of the rice root nematode Hirschmanniella oryzae on monsoon rice in Myanmar. Trop. Plant Pathol. 2010; 35, 3-10.

[7] MMA Youssef and MFM Eissa. The rice root nematode, Hirschmanniella oryzae, its identification, economic importance and control measures in Egypt: A review. Arch. Phytopathol. Pflanzenschutz 2014; 47, 2340-51.

[8] T Kyndt, D Fernandez and G Gheysen. Plant-parasitic nematode infections in rice: molecular and cellular insights. Annu. Rev. Phytopathol. 2014; 52, 135-53.

[9] DY Chen, HF Ni, JH Yen, RS Chen and TT Tsay. Distribution of rice root nematode Hirschmanniella oryzae and a new recorded H. mucronata (Nematoda: Pratylenchidae) in Taiwan. Plant Pathol. Bull. 2006; 15, 197-210.

[10] MLD Pascual, W Decraemer, I Tandingan De Ley, A Vierstraete, H Steel and W Bert. Prevalence and characterization of plant-parasitic nematodes in lowland and upland rice agro-ecosystems in Luzon, Philippines. Nematropica 2014; 44, 166-80.

[11] K Khun, W Decraemer, M Couvreur, G Karssen, H Steel and W Bert. Deceptive morphological variation in Hirschmanniella mucronata (Nematoda: Pratylenchidae) and a polytomous key to the genus. Nematology 2015; 17, 377-400.

[12] K Srimuang and P Ruanpanun. Surveillance of rice nematodes in rice field in Nakhon Nayok province (in Thai). In: Proceedings of $36^{\text {th }}$ Academic Conference on Rice and Cereals. Rice Department, Bangkok, Thailand. 2019, p. 138-45. 
[13] J Bridge, RA Plowright and D Peng. Nematode parasites of rice. In: M Luc, RA Sikora and J Bridge (Eds.). Plant parasitic nematodes in subtropical and tropical agriculture. CAB International, Wallingford, 2005, p. 87-130.

[14] JR Christie and VG Perry. Removing nematodes from soil. Proc. Helminthol. Soc. Wash. 1951; 18, 106-8.

[15] DL Coyne, JM Nicol and B Claudius-Cole. Practical plant nematology: A field and laboratory guide. $2^{\text {nd }}$ ed. SP-IPM Secretariat, International Institute of Tropical Agriculture (IITA), Cotonou, Benin, 2014, p. 31-48.

[16] M Holterman, A Wurff, S Elsen, H Megan, T Bongers, O Holovachov, J Bakker and J Helder. Phylum-wide analysis of SSU rDNA reveals deep phylogenetic relationships among nematodes and accelerated evolution toward crown clades. Mol. Biol. Evol. 2006; 23, 1792-800.

[17] EVD Berg, SA Subbotin, ZA Handoo and LR Tiedt. Hirschmanniella kwazuna sp. n. from South Africa with notes on a new record of H. spinicaudata (Schuurmans Stekhoven, 1944) Luc and Goodey, 1964 (Nematoda: Pratylenchidae) and on the molecular phylogeny of Hirschmanniella Luc and Goodey, 1964. Nematology 2009; 11, 523-40.

[18] M Suong, E Chapuis, V Leng, F Tivet, D Waele, HN Thi and S Bellafiore. Impact of a conservation agriculture system on soil characteristics, rice yield, and root-parasitic nematodes in a Cambodian lowland rice field. J. Nematol. 2019; 51, 1-15.

[19] G Besnard, N Thi-Phan, H Ho-Bich, A Dereeper, HT Nguyen, P Queneherve, J Aribi and S Bellafiore. On the close relatedness of two rice-parasitic root-knot nematode species and the recent expansion of Meloidogyne graminicola in Southeast Asia. Genes 2019; 10, 175.

[20] N Beesa, A Sasnarukkit, K Jindapunnapat, F Tivet, S Bellafiore and B Chinnasri. Species characterization and population dynamics of Hirschmanniella mucronata in lowland rice fields managed under conservation agriculture in Cambodia. J. Saudi Soc. Agr. Sci. 2021; 20, 137-45.

[21] D Peng, HS Gaur and J Bridge. Nematode parasites of rice. In: RA Sikora, D Coyne, J Hallmann and P Timper (Eds.). Plant parasitic nematodes in subtropical and tropical agriculture. $3^{\text {rd }}$ ed. CAB Publishing, Oxfordshire, 2018, p. 120-62.

[22] NC Burns. Soil pH effects on nematode populations associated with soybeans. J. Nematol. 1971; 3, 238-45. 